\title{
Tomografía computarizada 3D con técnica de A-Gi-VA CA II-Giacomotti-Vassaro]. Nuevo instrumento de díagnóstico de la estructura nasal
}

\section{A-GI-VA [Alí-Giacomotti-Vassaro]. A novel 3D computed tomography scan technique for diagnosis of the nasal structure}

Agustín H. ALI'*, Carlos CUTINI ${ }^{\star *}$, Juan C. GALLO ${ }^{\star * *}$

José D. GIACOMOTTI****, Víctor. O. VASSARO*

Resumen

Introducción y objetivo. La rinoplastia estética es uno de los procedimientos quirúrgicos más desafiantes, en donde converge una estructura tridimensional compuesta de tejido óseo, cartilaginoso y tegumentario, que debe ser interpretada y modificada como un todo para la obtención de un resultado óptimo.

La técnica A-GI-VA nos abre una ventana nueva para evaluar la anatomía nasal, principalmente los cartílagos nasales, sus relaciones entre sí y su disposición anatómica exacta, indispensable para el buen tratamiento de los mismos.

Material y método. Realizamos tomografías axiales computarizadas con reconstrucción tridimensional mediante tomógrafo helicoidal de múltiples cortes para analizar la estructura de los cartílagos nasales y su relación con la piel.

Resultados. Identificamos con precisión los componentes osteocartilaginosos de la nariz como ayuda en la planificación de la cirugía nasal.

Conclusiones. Mediantelautilización delatécnicaA-GI-VA podemos determinar con precisión la estructura nasal para su posterior clasificación y/o tratamiento.

\section{Palabras clave Rinoplastia, Tomografía computarizada,} Imagen tridimensional, Facial

\section{Nivel de evidencia científica Recibido [esta versión] Aceptado}

$5 c$ Diagnóstico

26 marzo/2019

26 noviembre/2019

Conflicto de intereses: Los autores declaran no tener ningún interés financiero relacionado con el contenido de este artículo. Financiación: No hubo fuentes externas de financiación para este trabajo.

* Cirujano Plástico, Práctica privada, Ciudad Autónoma de Buenos Aires, Argentina.

** Cirujano Plástico, Práctica privada, Bahía Blanca, Argentina.

*** Especialista en Diagnóstico por Imágenes, Sanatorio de Los Arcos, Ciudad Autónoma de Buenos Aires, Argentina.

**** Cirujano Plástico, Universidad de Buenos Aires, Ciudad Autónoma de Buenos Aires, Argentina.

Background and objective. Aesthetic rhinoplasty is one of the most challenging surgical procedures, where a bony and a cartilaginous framework covered by skin meet into a tridimensional structure that must be interpreted and modified as one to obtain a successful result.

Methods. A regular 3D CT scan of the maxillofacial area with shaded surface display (SSD) technique is used to get a precise evaluation of the nasal cartilages and their relationship with the skin.

Results. We obtained a greater detail of the nasal structure, both of its components: bone cartilage in order to improve nasal surgery planning.

Conclusions. The A-GI-VA technique allows us to depict the nasal anatomy and determine a better surgery planning.

Key words Rhinoplasty, Computed tomography, Tridimensional images, Facial

\section{Level of evidence Received [this version] \\ Accepted}

$5 c$ Diagnostic

$26 \mathrm{Marzo} / 2019$ 26 November/2019 


\section{Introducción}

La rinoplastia estética es uno de los procedimientos quirúrgicos más desafiantes, en el que convergen una estructura tridimensional compuesta de tejido óseo, cartilaginoso y tegumentario, que debe ser interpretada y modificada como un todo para la obtención de un resultado óptimo. Debido a esta compleja interrelación de componentes, la comprensión de la anatomía nasal que subyace a la cubierta tegumentaria determinará la guía para una ejecución quirúrgica adecuada.

Hoy en día, la Medicina cuenta con muchos instrumentos para el estudio y evaluación de los pacientes. Los estudios tomográficos visualizan principalmente las estructuras óseas, pero ninguno se aboca de forma específica a las estructuras cartilaginosas y a las partes blandas. El único componente cartilaginoso que se eva-

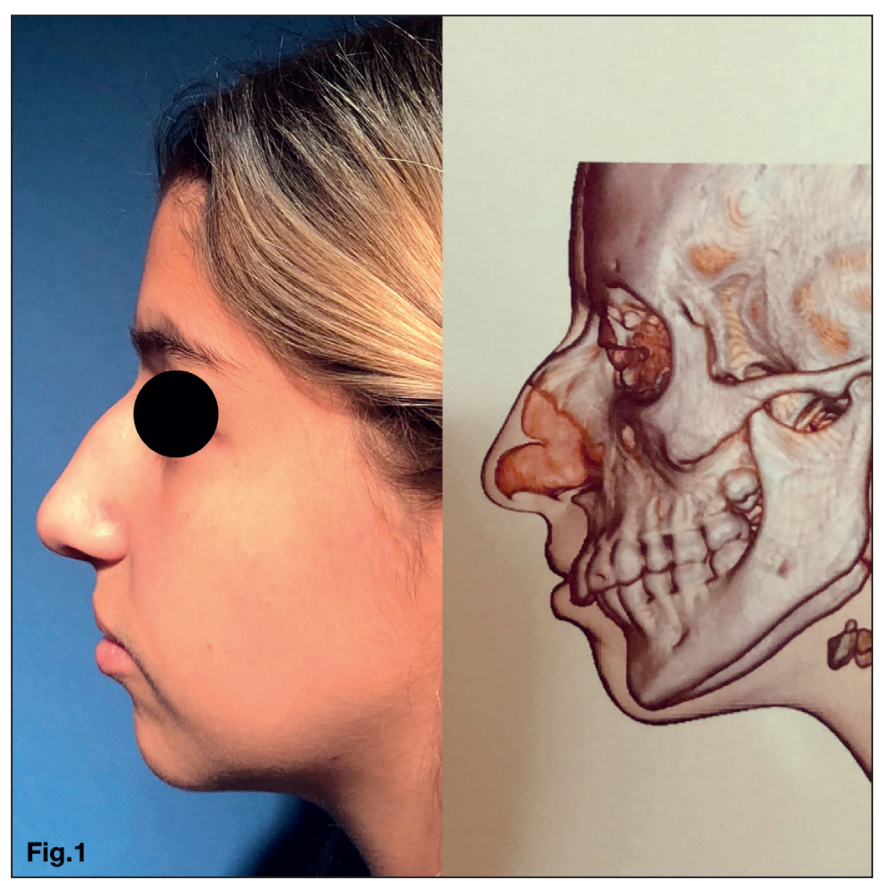

Fig 1. Se puede determinar el componente osteocartilaginoso de la giba dorsal y su relación con la punta nasal.

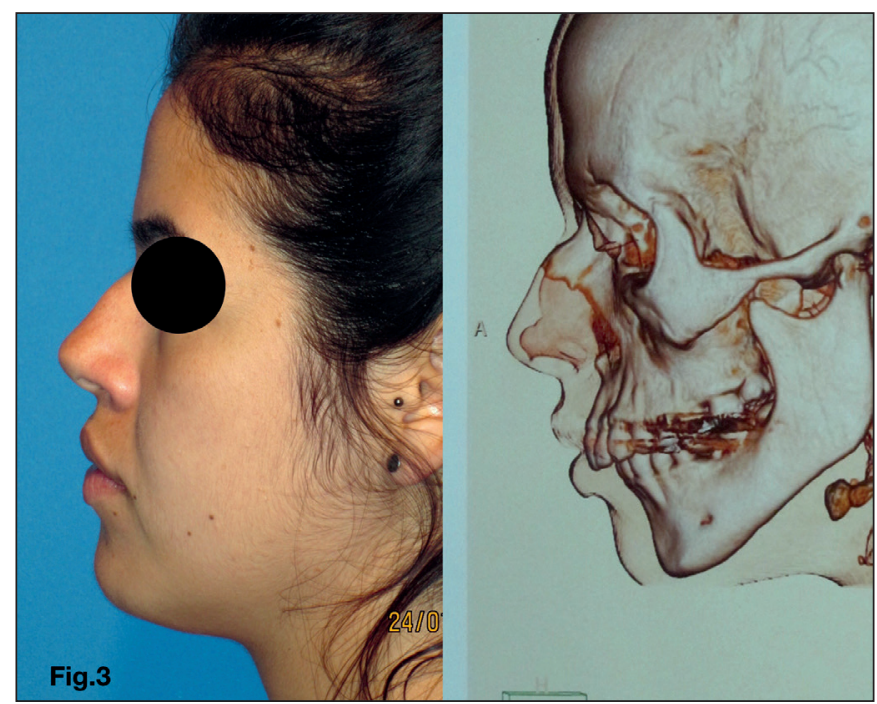

Fig 3. Deformidad nasal preponderantemente ósea a nivel de la giba dorsal. lúa en forma rutinaria con la tomografía computarizada es el cartílago cuadrangular, cuando se profundiza en el examen del septum nasal. Hasta el momento, las publicaciones de estudios complementarios que se utilizan para optimizar la evaluación de las estructuras nasales están focalizadas en la radiografía y/o en la tomografía para la detección y clasificación de fracturas nasales, ${ }^{(1,2)}$ para la valoración del septum nasal y de los cornetes en la identificación etiológica de las obstrucciones aéreas nasales $^{(3-6)}$ y en la interpretación indirecta de las alteraciones cartilaginosas mediante la comparación de fotografías. ${ }^{(7-10)}$

Consideramos que todos los elementos que constituyen la pirámide nasal afectan en menor o mayor medida a la forma definitiva de la nariz a tratar. También sabemos que las relaciones y proporciones que tienen las estructuras osteocartilaginosas entre sí, nos van a dar como

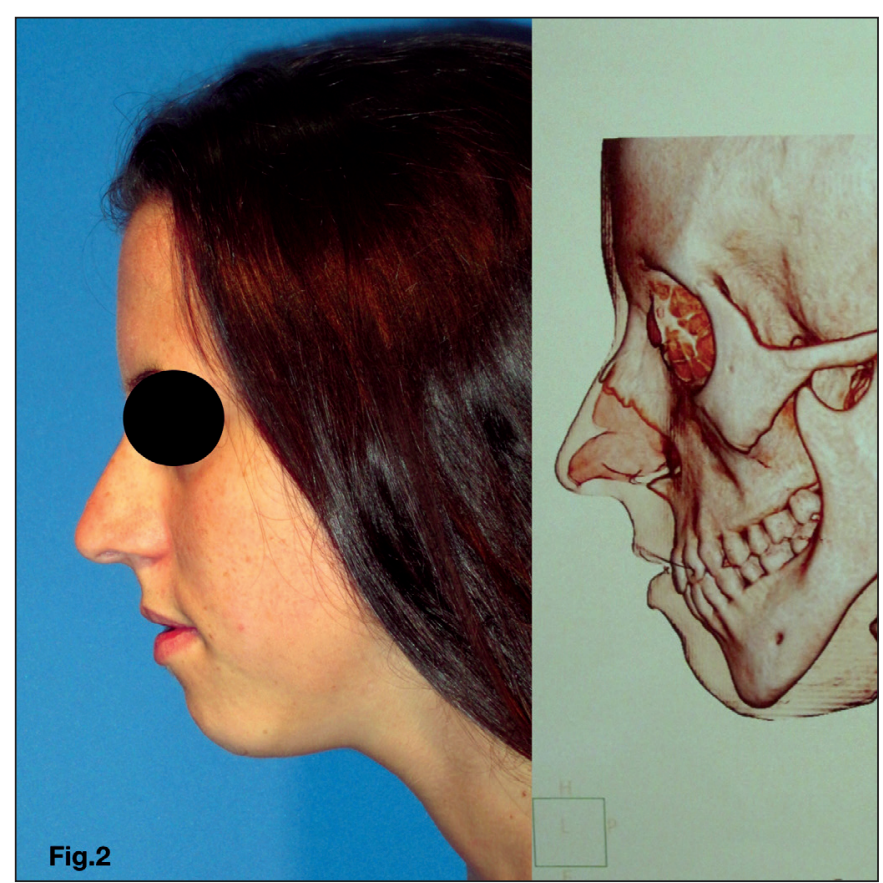

Fig 2. Mujer con giba nasal de predominio óseo, con un resultado no armónico en relación a la punta nasal.

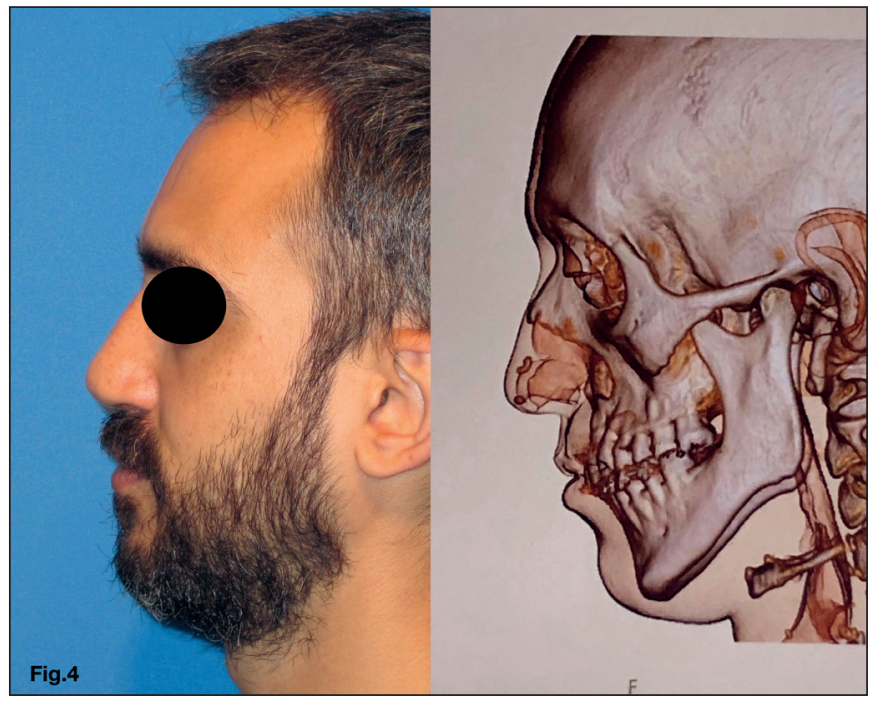

Fig 4. Presencia de cartílagos sesamoideos en paciente con nariz leporina. 

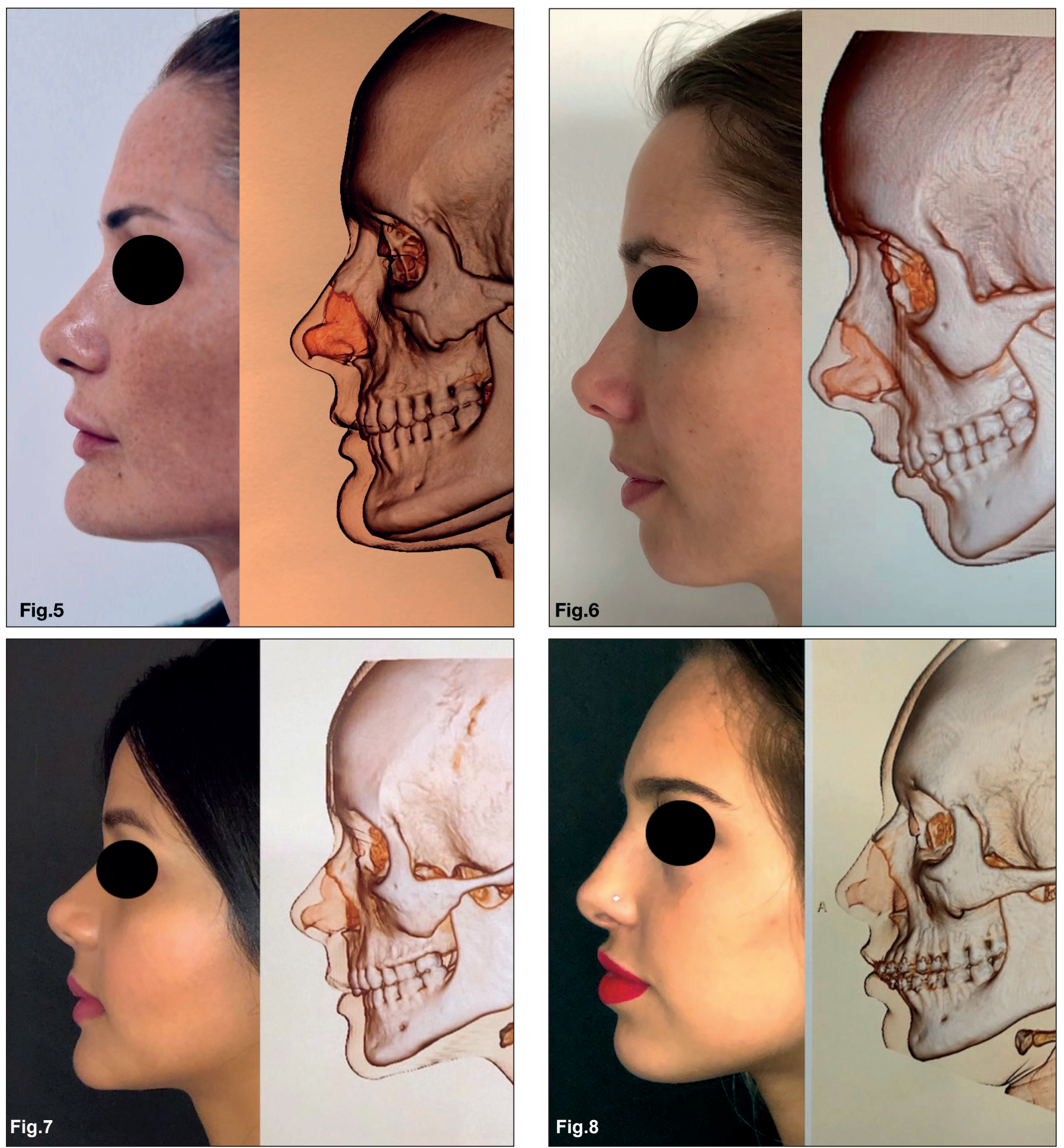

Figuras 5 a 8. Pacientes con perfiles nasales estéticamente armónicos que no fueron sometidos a rinoplastia.

resultado una nariz bella proporcionada o desproporcionada; es por eso que si podemos objetivar con precisión el tamaño, las relaciones y proporciones de todos los elementos a tratar, podremos planificar una rinoplastia con un resultado más exitoso y predecible al saber con mayor precisión qué tratamiento debemos realizar sobre cada uno de los elementos en cuestión.

La tomografía con reconstrucción 3D no nos da una visualización perfecta de todos los elementos de la estructura nasal, sino solo de los elementos óseos. El objetivo de este trabajo radica en mostrar un instrumento más en la evaluación del paciente candidato a una rinoplastia con una herramienta de uso cotidiano, como es el tomógrafo computarizado.

\section{Material y método}

La tomografía computarizada (TC) con reconstrucción 3D es un estudio por imagen de uso habitual en nuestro medio. Para la ejecución de la técnica A-GI-VA, nombre que deriva de las iniciales de los apellidos de los cirujanos que la crearon, Alí-Giacomotti-Vassaro, se 
requiere un equipo de $\mathrm{TC}$ con la suficiente potencia para poder trabajar con gráficos en alta definición, aplicado al estudio de la estructura nasal.

La técnica que empleamos para poder generar este tipo de imagen se denomina: "renderización de superficie (SR)" o "renderización de superficie sombreada". Se entiende por el término "renderización" la utilización de herramientas digitales que generan una imagen o foto realista desde un modelo tridimensional a un modelo bidimensional (como el que se pude obtener en un hoja de papel). Para conseguir una sensación de perspectiva o tridimensionalidad (3D), se realiza un sombreado de superficie (imagen en cuestión) y se genera un efecto 3D.

En el caso puntual de la técnica A-GI-VA, utilizamos filtros de imagen predeterminados que se hallan en el software del TC, y que en nuestro caso desarrollamos utilizando un tomógrafo Phillips Brillance $64^{\circledR}$, si bien los nombres de los filtros son universales y se extienden a todos los software de los diferentes tomógrafos modernos.

Para reproducir la técnica A-GI-VA, después de realizar una TC del macizo craneofacial del paciente con su componente óseo, se debe aplicar el filtro denominado "Aorta 1 trans" y luego modificar los rangos de densidades de la imagen tomográfica, acción a la que comúnmente se denomina "ventanear", hasta que queden en evidencia los cartílagos nasales triangulares, alares y cuadrangular. Al mismo tiempo nos permite observar la exteriorización a nivel cutáneo de los accidentes que reproducen dichos componentes.

\section{Resultados}

Realizamos este estudio desde el año 2015, tanto en pacientes que son candidatos a rinoplastia como en pacientes que, previo consentimiento, presentan una nariz estéticamente armónica y nos permiten su estudio. Hasta el momento llevamos aplicada esta técnica en 356 pacientes.

Con la técnica A-GI-VA identificamos con precisión todos los componentes de la nariz en la evaluación preoperatoria (Fig. 1-4), lo cual nos permitirá valorar y medir:

- La altura y trayecto de los huesos propios.

- La altura y trayecto de los cartílagos triangulares.

- La altura y disposición de los cartílagos alares (indispensable para el correcto tratamiento de la punta y del dorso nasal).

A su vez, esta técnica también permite observar e identificar, a modo comparativo, todas las estructuras mencionadas en pacientes que no han sido intervenidos quirúrgicamente (Fig. 5-8) y presentan una nariz estéti- camente armónica. Vemos así las relaciones y proporciones de los cartílagos nasales y los huesos propios, como también la forma resultante que estas estructuras determinan sobre la cobertura tegumentaria.

Las ventajas de la TC con reconstrucción tridimensional con técnica A-GI-VA son, a nuestro modo de ver:

1- Evaluar y medir el hueso propio.

2- Evaluar y medir el cartílago triangular.

3- Evaluar y medir el cartílago alar en sus pars medial y lateral.

4- Evaluar el contorno cutáneo nasal.

5- En rinoplastias secundarias, evaluar previamente las estructuras nasales para planificar la cirugía con mayor precisión.

6- Evaluar las deformidades nasales, como el componente estructural remanente, y planificar los tamaños de las resecciones o reconstrucciones que debemos realizar.

7- En el postoperatorio permite evaluar la disposición de los elementos, si fue alterada o no.

8- En caso de utilizar injertos se puede ver la evolución de los mismos, su disposición y permanencia.

9- Identificar y caracterizar la anatomía nasal del paciente, permitiendo su clasificación de forma objetiva $\mathrm{y}$ en un modo no invasivo.

Discusión

Consideramos que todos los elementos que constituyen la pirámide nasal afectan en menor o mayor medida a la forma definitiva de la nariz a tratar. También sabemos que las relaciones y proporciones que tienen las estructuras osteocartilaginosas entre sí, nos van a dar el resultado de una nariz bella proporcionada o desproporcionada. Esto ya fue demostrado por Giacomotti y col. en su trabajo sobre anatomía nasal realizado en cadáveres y publicado en esta misma revista. ${ }^{(11)}$ Es por ello que, si podemos objetivar con precisión el tamaño, las relaciones y proporciones de todos los elementos a tratar, creemos que podemos planificar una rinoplastia con un resultado exitoso y predecible. Es ahí donde la técnica A-GI-VA nos ofrece una nueva metodología a la hora de planificar una rinoplastia estética.

En nuestro estudio nos planteamos la hipótesis de que esta técnica de imágenes, aplicada a pacientes que ya fueron sometidos a rinoplastia, nos podría ser de gran utilidad para ayudarnos en una mejor interpretación de la anatomía nasal en pacientes candidatos a rinoplastias secundarias o terciarias, y a la evaluación de sus resultados postoperatorios.

El costo de la TC con técnica A-GI-VA es el mismo que el de una $\mathrm{TC}$ con reconstrucción $3 \mathrm{D}$, ya que se utiliza 
un tomógrafo computarizado multicorte y el software de serie del mismo, lo que también es un factor adicional a considerar, dada su utilidad sin sobrecosto para los pacientes.

Hasta el momento, esta es la primera publicación de una serie en las que pretendemos utilizar la técnica A-GIVA para la clasificación de las narices basándonos en sus alteraciones osteocartilaginosas y tomando como guía la clasificación propuesta por Giacomotti. ${ }^{(11)}$

\section{Conclusiones}

La TC con reconstrucción tridimensional con técnica A-GI-VA permite una mejor interpretación de la estructura nasal y de su cobertura cutánea, favoreciendo una planificación quirúrgica más precisa y sin implicar un coste adicional, más allá del propio de una TC 3D convencional.

\section{Dirección del autor}

Dr. Agustín Hugo Alí

Av. Santa Fe $32691^{\circ}$

Ciudad Autónoma de Buenos Aires, Argentina.

Correo electrónico: aali@intramed.net

\section{Bibliografía}

1- Han DS, Han YS, Park JH. A new approach to the treatment of nasal bone fracture: radiologic classification of nasal bone fractures and its clinical application. J Oral Maxillofac Surg. 2011;69(11):2841-2847.

2- Nigam A, Goni A, Benjamin A, Dasgupta AR. The value of radiographs in the management of the fractured nose. Arch Emerg Med. 1993;10(4):293-297.

3- Karataş D, Yüksel F, Şentürk M, Doğan M. The contribution of computed tomography to nasal septoplasty. J Craniofac Surg. 2013;24(5):1549-1551.

4- Kassel EE, Cooper PW, Kassel RN. CT of the nasal cavity. $J$ Otolaryngol. 1983;12(1):16-36.

5- Ardeshirpour F, McCarn KE, McKinney AM, Odland RM, Yueh B, Hilger PA. Computed tomography scan does not correlate with patient experience of nasal obstruction. Laryngoscope. 2016;126(4):820-825.

6- Andrades P, Cuevas P, Danilla S, Bernales J, Longton C, Borel C, Hernández $\mathbf{R}$, Villalobos $\mathbf{R}$. The accuracy of different methods for diagnosing septal deviation in patients undergoing septorhinoplasty: A prospective study. J Plast Reconstr Aesthet Surg. 2016;69(6):848-855

7- Kayabasoglu G, Nacar A. The Soft Triangle: An Often Neglected Area in Rhinoplasty. Aesth Plast Surg. 2015;39(5):659-666.

8- Kluba S, Bopp C, Bacher M, Reinert S, Krimmel M. Morphological analysis of the lip and nose following cleft lip repair with simultaneous partial primary rhinoplasty: A prospective study over 4 years. J Craniomaxillofac Surg. 2015;43(5):599-605.

9- Agarwal R, Chandra R. Alar web in cleft lip nose deformity: study in adult unilateral clefts. J Craniofac Surg. 2012;23(5):1349-1354.

10- Saleh MA, Elshahat A, Emara M, Hussein H, Gould L, Smith D Jr, Awad MA. Objective tools to analyze the lower lateral cartilage in unilateral cleft lip nasal deformities. J Craniofac Surg. 2011;22(4):1435-1439.

11- Giacomotti, José D., Ali, Agustín, López, Tomás, \& Piqué, Héctor. (2016). Anatomía del dorso nasal: estudio del tercio inferior en la nariz naturalmente armónica. Cir plást. iberolatinoam. 2016; 42(4), 313-320. 
